(C)2010 IEEE. Personal use of this material is permitted. However, permission to reprint/republish this material for advertising or promotional purposes or for creating new collective works for resale or redistribution to servers or lists, or to reuse any copyrighted component of this work in other works must be obtained from the IEEE. 


\title{
An Optimal Parameter Estimation Method for Soft Tissue Characterization
}

\author{
Yashar Madjidi, Yongmin Zhong, Bijan Shirinzadeh, Julian Smith
}

\begin{abstract}
This paper presents a gradient-free direct search estimation method by using genetic algorithm to model and predict the elastic stress response of ligament based on quasi-linear viscoelastic (QLV) theory. An improved genetic algorithm is developed to simultaneously fit the ramping and relaxation experimental data to the QLV constitutive equation for obtaining soft tissue parameters in a time-saving process. Experiments and comparison analysis with the existing methods for two exponential and polynomial QLV models are conducted, demonstrating that the proposed method can accurately estimate soft tissue parameters and satisfy the time-saving requirement of intraoperative soft tissue characterization.
\end{abstract}

\section{INTRODUCTION}

$\mathrm{S}$ OFT tissue properties are important to many modern applications of technology to medicine, such as robotic surgery, soft tissue modelling and surgical simulation with force feedback. However, realistic acquisition of soft tissue properties is extremely challenging not only because of the non-linearity, rate, and time-dependence of soft tissues, but also due to the layered and non-homogeneous structures of soft tissues [1-7]. It is understood that soft tissue properties may dynamically change during the surgical process according to different patients, different organs, different functional regions and layers crossed by the surgical tools, and different physiological conditions. Therefore, it requires that mechanical properties of soft tissues be acquired and studied through a time-saving intraoperative measurement process. Currently, soft tissue properties are commonly acquired by conducting standard mechanical testing such as tensile and indentation tests on material samples extracted from organs under well-defined loading and boundary conditions [8,9]. Since the thickness and transversal area of organs are not easy to measure, strain

Manuscript received March 20, 2010. This work was supported by the ARC (Australian Research Council) Discovery project (project number: 0986814).

Y. Madjidi is with the Department of Mechanical and Aerospace Engineering, Monash University, Clayton, VIC 3800, Australia. (e-mail: ymadjidi@gmail.com).

Y. Zhong is with the Department of Mechanical Engineering, Curtin University of Technology, GPO Box U1987, Perth, WA 6845, Australia (corresponding author to provide phone: 61-8-92667657; fax: 61-892662681; e-mail: y.zhong@urtin.edu.au).

B. Shirinzadeh is with Department of Mechanical and Aerospace Engineering, Monash University, Clayton, VIC 3800, Australia. (e-mail: Bijan.Shirinzadeh@eng.monash.edu.au).

J. Smith is with the Department of Surgery, Monash Medical Centre, Monash University, Clayton, VIC 3800, Australia. (e-mail: Julian.Smith@med.monash.edu.au). and stress values cannot be obtained directly from the measured displacement and force data [6, 10]. Tissue properties in vitro are available from a number of sources $[11,12]$. However, since such experimental conditions are difficult to achieve, the parameters estimation methods based on in-vitro experiments may not be widely used. Given the difficulty of experimental approaches for online soft tissue characterisation, it is necessary to study an analytical solution for accurately predicting the mechanical behaviours of soft tissues and satisfying the time-saving requirement of intra-operative measurement.

There have been only a few methods focusing on studying the ability of the quasi-linear viscoelastic (QLV) model for estimating the overall stress-strain responses of ligaments and tendons [10, 13, 14], although this model has been widely used to characterize soft tissue behaviours [15]. These approaches are mainly based on regression algorithms to find a gradient-based solution, rather than gradient-free optimization algorithms, for characterization of soft tissue properties. The gradient based algorithm has a fast convergence by using the derivatives with respect to the objective function. However, it is difficult to handle discontinuous and non-differentiable problems and also involves the variability of constants [16]. Alternatively, gradient-free methods are typically designed to solve optimization or approximation problems whose objective function is computed by a black-box. Especially when the gradient computation is unavailable or expensive in aspect of speed/time and accuracy, estimating derivatives by finite differences may be prohibitively costly. Among gradientfree algorithms, genetic algorithm (GA) can be applied to solve a variety of optimization problems that are specifically not well suited for standard optimization algorithms, including problems in which the objective function is discontinuous, non-differentiable, stochastic, or highly nonlinear [17]. GA is also suitable for a large number of quantized parameters and is less susceptible to getting stuck at local optima.

Currently, the existing studies focusing on accurate and time-saving soft tissue characterization are mainly dominated by explicit FEM [6]. There has been very limited research focusing on using GA for soft tissue characterization. Kohandel et al reported a genetic algorithm to estimate the QLV model parameters [18]. However, this algorithm is at a preliminary stage, only providing with simple results to verify the curve matching ability. Chawla et al reported a method by combining GA with inverse finite element analysis to study the characterization of human passive muscles under impact loads to identify the dynamic 
material properties for strain rates relevant to automobile crashes [10]. However, the GA is only used to minimize the differences between experimental and finite-element force responses.

This paper focuses on establishing a gradient-free method for estimation of the QLV model parameters, rather than a gradient-based approach in the previously existing studies. It investigates the estimation ability of a direct search tool to calculate and predict the parameters of soft tissues for achieving optimum intra-operative characterization. It establishes an improved time-saving GA as a gradient-free direct search tool to estimate and predict the QLV parameters explicitly without approximating their derivatives. Experiments and comparison analysis with an exponential formulation model [19] and a polynomial form of the Mooney-Rivlin (MR) model [20] have been conducted to comprehensively evaluate the performance of the proposed method. Since extensive theories and experiments demonstrate that the robustness of gradient-free optimization methods does not suffer from the cases with a moderate level of noise, it is reasonable that the experimental analysis is conducted under an assumption that no noise is existed.

\section{BIOMECHANICAL MODELS}

\section{A. QLV Model}

The QLV model has become the most widely used theory in the biomechanics of soft tissues [14]. The QLV theory combines elastic and time-dependence components of a tissue's mechanical response by using an integral formulation. The basic idea of this theory is that the stress at a given time can be described by a convolution integral representation to separate the elastic response and the relaxation function, and the relaxation function has a specific continuous spectrum. According to the QLV theory, the complete stress in a tissue subjected to a step strain can be expressed by the following convolution formula

$$
\sigma(t)=G(t) * \sigma^{e}(\lambda)
$$

where $G(t)$ is the reduced relaxation function, $\sigma^{e}(\lambda)$ is the nonlinear elastic response, and $\lambda(t)$ is the stretch ratio. In general, $G(t)$ is a fourth-order tensor for describing direction-dependent relaxation phenomena.

Using the Boltzmann superposition principle and representing the strain history as a series of infinitesimal step strains, the overall stress relaxation function can be expressed as the sum of all individual relaxations. For a general strain history, the stress at time $t$ is given by the convolution integral of $G(t)$ over time

$$
\sigma(t)=\int_{-\infty}^{t} G(t-\tau) \cdot \frac{\partial \sigma^{e}(\lambda)}{\partial \lambda} \cdot \frac{\partial \lambda}{\partial \tau} \cdot d \tau
$$

where $\partial \sigma^{e}(\lambda) / \partial \lambda$ represents the instantaneous elastic response, and $\partial \lambda / \partial \tau$ is the stretch history. For biological soft tissues, it is commonly assumed that the relaxation function is the same and continuous in all directions, and thus $G(t)$ can be simplified as a scalar

$$
G(t)=\frac{1+c \cdot\left[E_{1}\left(t / \tau_{1}\right)-E_{1}\left(t / \tau_{2}\right)\right]}{1+c \cdot \ln \left(\tau_{2} / \tau_{1}\right)}
$$

where $E_{1}(y)=\int_{y}^{\infty}\left(e^{-z} / z\right) \cdot d z$ is the exponential integral function, $\tau_{1}$ and $\tau_{2}$ represent time constants that bind the lower and upper limits of the constant damping range for the relaxation function, and $c$ is a dimensionless constant that scales the degree such that viscous effects are occurred. These three viscoelastic material coefficients can be determined from the analysis of a stress relaxation experiment.

For the purpose of integrability and time-saving calculation, the temporal behaviours of the relaxation function is described by a decaying exponential equation

$$
G(t)=a e^{-b t}+c e^{-d t}+g e^{-h t}
$$

where coefficients $a, c$ and $g$, and exponents $b, d$ and $h$ are constants to be either determined experimentally or estimated numerically.

\section{B. Mooney-Rivlin Model}

The MR model has been widely considered to estimate the elastic stress response of soft tissues in biomechanics [20, 21]. In this model, the strain energy function may be expressed in terms of the principal invariants $I_{1}$ and $I_{2}$ of the right Cauchy-Green tensor $\mathbf{C}$ as

$$
\begin{gathered}
W=C_{1}\left(I_{1}-3\right)+C_{2}\left(I_{2}-3\right) \\
I_{1}=\operatorname{Tr}(\mathbf{C})=\lambda_{1}^{2}+\lambda_{2}^{2}+\lambda_{3}^{2} \\
I_{2}=\frac{1}{2}\left[\operatorname{Tr}(\mathbf{C})^{2}-\operatorname{Tr}\left(\mathbf{C}^{2}\right)\right]=\lambda_{1}^{2} \cdot \lambda_{2}^{2}+\lambda_{1}^{2} \cdot \lambda_{3}^{2}+\lambda_{2}^{2} \cdot \lambda_{3}^{2}
\end{gathered}
$$

where $\lambda_{1}, \lambda_{2}$ and $\lambda_{3}$ represent the principal stretches, and $C_{1}$ and $C_{2}$ are the material constants [20]. The principal Cauchy stresses for an incompressible and isotropic material in the case of uniaxial tension can be calculated by

$$
\sigma^{e}=2\left(\lambda^{2}-1 / \lambda\right) \cdot\left(C_{1}+C_{2} / \lambda\right) ; \quad C_{1}+C_{2}>0
$$

where $\sigma^{e}$ and $\lambda$ are the stress and stretch in the axial direction, respectively. The axial stretch can be converted to 
the engineering strain by

$$
\varepsilon=\frac{\Delta L}{L}=\lambda-1
$$

where $L$ is the length of the specimen and $\Delta L$ is the change of the length.

\section{Exponential Formulation}

Exponential formulation has been used in soft tissue models for describing the tensile behaviors of ligaments and tendons. One of the most widely used exponential expressions is an empirical equation, which was firstly proposed for the tensile behavior of skin

$$
\sigma^{e}=A\left(e^{B \varepsilon}-1\right) ; \quad A \& B>0
$$

where $A$ and $B$ represent the material constants, which are determined by fitting the model to the experimental data. However, this model does not consider 3-dimensional stress states, and is not generally expressed in terms of a strain energy function. Although there are other models based on exponential formulations reported in the literature, these models are not able to accurately predict elastic stress strain response for the tensile behavior of ligaments. Hence, they are not considered in this study for the comparison purpose.

\section{Proposed GenetiC ALGORITHM}

The GA uses three main types of rules at each step to create the next generation from the current population: selection rules choosing the individuals, called parents, which contribute to the population at the next generation; crossover rules combining two parents to form children for the next generation; and mutation rules applying random changes to individual parents to form children.

\section{A. Fitness Function}

The population members are ranked on the basis of fitness function and then their ranks are typically divided by the number of individuals to provide a probability threshold for the selection. Scaling is another factor considering the recent history of the population and assigning fitness values based on the comparison of individual performance to the recent average performance of the population. Fitness values can be assigned based on their actual distances from the floor value, or can be equally spaced for the purpose of simplicity.

In this paper, the relaxation function (4) and the elastic model (8) are used in the QLV model (2). Considering engineering strain over the ramping period $\left(0<t<t_{0}\right)$, the stress resulted from a ramp phase with a constant strain rate $\gamma$ may be written as

$$
\begin{aligned}
& \sigma\left(t: 0<t<t_{0}, \theta\right)= \\
& 2 \gamma \int_{0}^{t}\left(a e^{-b(t-\tau)}+c e^{-d(t-\tau)}+g e^{-h(t-\tau)}\right) \\
& \quad\left(2 C_{1}(\gamma \tau+1)+\frac{C_{1}(\gamma \tau+1)+2 C_{2}}{(\gamma \tau+1)^{3}}+C_{2}\right) d \tau
\end{aligned}
$$

and the stress response from $t_{0}$ onwards, where the strain rate is zero, is simply left with

$$
\begin{aligned}
& \sigma\left(t: t \geq t_{0}, \theta\right)= \\
& 2 \gamma \int_{0}^{t_{0}}\left(a e^{-b(t-\tau)}+c e^{-d(t-\tau)}+g e^{-h(t-\tau)}\right) \\
& \quad\left(2 C_{1}(\gamma \tau+1)+\frac{C_{1}(\gamma \tau+1)+2 C_{2}}{(\gamma \tau+1)^{3}}+C_{2}\right) d \tau
\end{aligned}
$$

where $\theta=\left[C_{1}, C_{2}, a, b, c, d, g, h\right]$ is the vector of constants to be estimated by using the proposed GA.

For a set of experimental data, the ramping portion of the data is defined as $\left(t_{i}, \mathbf{R}_{i}\right)$ for $0<t<t_{0}$ and the relaxation data as $\left(t_{i}, \mathbf{S}_{i}\right)$ for $t_{0}<t<\infty$ [22]. Thus, the sum of square of the differences between the experimental and theoretical data is

$$
f(\theta)=\sum_{i}\left[\mathbf{R}_{i}-\sigma\left(t_{i}: 0<t_{i}<t_{0}, \theta\right)\right]^{2}
$$

and

$$
g(\theta)=\sum_{i}\left[\mathbf{S}_{i}-\sigma\left(t_{i}: 0<t_{i}<t_{0}, \theta\right)\right]^{2}
$$

Both (13) and (14) are a function of $\theta$. The objective is to minimize these two functions simultaneously by using the proposed GA. Hence, the sum of the two functions is considered as the fitness function

$$
\text { Fitness function }=f(\theta)+g(\theta)
$$

\section{B. Population}

The initialization of the population is usually performed stochastically. Regardless of the process used, it requires that the population represent a wide assortment of individuals. The main trade-off on the size of populations is obvious, i.e. a large population searches the space more completely, but at a higher computational cost. Further, although the population size tends to increase with the individual string length linearly, rather than exponentially, the optimal population size also depends on the problem. In this study, an initial size of population $n=200$ was firstly 
chosen and the best individuals did have the highest fitness of all possibilities when the population convergence was occurred. To satisfy the time-saving requirement of intraoperative soft tissue measurement, the initial size of population $n=100$ was finalized according to the required precision for the fitness function.

\section{Selection}

The selection function chooses parents for the next generation based on their scaled values from the fitness scaling function. The main three widely used selection schemes are roulette wheel implementation, tournament selection and elitism. With the standard roulette wheel selection, the individual with the highest fitness value in a given generation may not survive reproduction, crossover and mutation, and thus being unaltered in the new generation. Tournament selection selects each parent by randomly considering the size of tournament players (individuals) to obtain the best individual (the one with higher fitness). This study chooses tournament selection as the selection rule, and the size of tournament players for choosing individuals is set to two.

\section{Crossover}

The most effective operator in GA is crossover based on the metaphor of sexual combination. Crossover has two main attributes, which can be varied. One is the probability of occurrences, and the other is the type of crossover to be implemented. The most basic crossover type is one-point crossover, which involves selecting a single crossover point at random and exchanging the portions of the individual strings to the right of the crossover point. Two-point crossover with a probability of $0.60-0.80$ is a relatively common choice for another type of crossover. Uniform crossover is also a useful crossover type, in which a random decision is made at each bit position in the string according to whether the bits between parent strings are exchanged or not. Uniform crossover sometimes works better with slightly lower crossover probability. It is also common to start out running the GA with a relatively higher value for crossover, then taper off the value linearly to the end of the computational trial, ending with a value of one half or two thirds of the initial value [17]. In this study, considering the significant influence of crossover function and its fraction value on the convergence performance of GA, the behavior of the latter method was verified through different functions and fractions. Consequently, single point function with less than 60 percent of the fraction value was chosen for the proposed problem.

\section{E. Mutation}

Mutation is the stochastic flipping of bits occurring at each generation, which is often held constant for the entire computational trial of the GA. In this study, the fixed rate of 0.01 was defined for the mutation process and the change of the mutation rate during the computational trials was not hypothesized as an influential operator.
As a matter of fact, mutation is not an especially important operator in GA and is usually set at a very low rate, or sometimes omitted altogether. Traditional crossover and mutation operators are based on a randomization mechanism. However, improved GAs generally include new adaptive penalty schemes and adaptive mutations as well as adaptive crossover operators. In this study, a penalty factor higher than common values was used. Especially, setting a higher value for the initial penalty after randomization of initial population was observed as a noticeable factor on the GA convergence performance.

\section{PERFORMANCE ANALYSIS}

A prototype system has been implemented with the proposed method for characterization of soft tissue properties in a time-saving process. Experiments have been conducted to examine the ability of the proposed method to predict the elastic stress relaxation behavior. Since the MR model and exponential formulation are commonly used for the prediction and analysis of soft tissue responses, the comparison analysis has been conducted with respect to other existing methods using these two models.

Among soft biological tissues, tendons and ligaments play an important role in the mechanical integrity of the body by transferring loads between bones for the ligaments, or between muscles and bones for the tendons. Anterior cruciate ligament (ACL) is one of the major ligaments of the knee. It has been used in a great number of both experimental and computational studies. Since ACL has been used as a popular subject and the estimated and predicted QLV models based on the same experimental data have been available in the literature [14], experiments have been conducted by using the same data to evaluate the performance of the proposed approach for the convenience of comparison analysis.

In derivation of the constitutive equation, we need to choose appropriate models for the instantaneous elastic response and the relaxation function. The exponential formulation (10) has been studied for tendons and ligaments [8]. The MR formulation and Ogden formulation have been widely used for elastic and hyperelastic soft tissues such as brain [23]. The relaxation functions based on (3) and (4) are also widely used by assuming a continuous relaxation spectrum [9]. In this study, in order to predict the stress relaxation response of the ACL, the elastic models (exponential and MR formulations) (8) and (10) together with the decaying exponential equation (4) for the relaxation function are used in the QLV model (2). The related experimental data are derived from the previously reported and investigated data sets $[15,19,24]$. Three different QLV models are investigated in this study by using both a gradient-based and GA approach. Two of the QLV models comprise two elastic models and the exponential relaxation function (4). The parameters of these two models are estimated through a gradient-based method by fitting the elastic model and the relaxation function separately under 
the assumption that the load is applied instantaneously. The third QLV model, which is the main investigated QLV model in this study, comprises the MR elastic model and the exponential relaxation function. The parameters of this QLV model are estimated by using the GA as a gradient-free method. Similar to the previous studies [18, 22], the ramping and relaxation portions are simultaneously fitted to the constitutive equation in order to remove the assumption of a step change in strain. All the models are first fitted to the stress relaxation data measured at the $2 \%$ strain level to determine the material constants. These models are then used to predict stress relaxation at higher strain levels.

Experiments have been conducted to use the models to fit the stress-relaxation data for ACL. As shown in Fig. 1(a), it can be seen that all of the models closely match the experimental data. During the approximation process based on the GA, the trend of curve matching can be tracked through generations by determining its behaviours based on the fitness function value and average distance between individuals as well as the curve matching ability to fit the experimental data. The GA satisfies the time-saving requirement of intra-operative measurement to determine the QLV parameters. The convergence ability of this method can be adjusted according to the required precision by treating the fitness function limit as one of the stopping criteria.

Experiments have also been conducted to evaluate the time-saving factor in aspect of curve matching quality. One investigation is focused on defining the weight coefficient around the change point of data portion from the ramping phase to the relaxation phase of the diagram. The other is focused on defining the appropriate slope for the relaxation phase in accordance with the time-dependent reduction of stress at the end of the time period (1600s). Experiments show that these two techniques can get the desired curve matching with fewer generations. However, the latter solution tends to obtain the optimally estimated QLV parameters with fewer generations in comparison with the former solution. It should also be noted that the estimation of upper and lower bounds for starting the search algorithm is also an effective way to achieve a faster convergence. With the ability provided by these techniques and adjusting the optimal fitness function limit, the proposed GA provides a systematic gradient-free solution for intra-operative measurement to determine the QLV parameters during a reasonable time period.

After verifying the QLV models with the stress relaxation data measured at the $2 \%$ strain level to determine the material constants, these models can be used to predict the stress relaxation response of ACL at the $4 \%$ and $6 \%$ strain levels and performing comparison analysis with the experimental data. Although all the models closely match the experimental data at the $2 \%$ strain (Fig. 1(a)), the prediction ability of the models at higher strain levels is limited. Fig. 1(b) and Fig. 1(c) generally indicate that higher strain levels cause more overestimated stress response especially in a longer relaxation time, in comparison with the strain level curve matched to determine the QLV parameters.

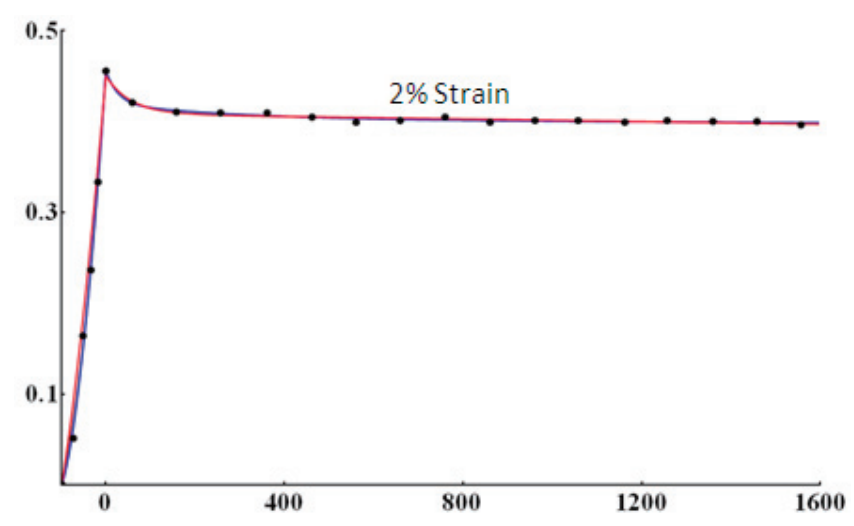

(a)

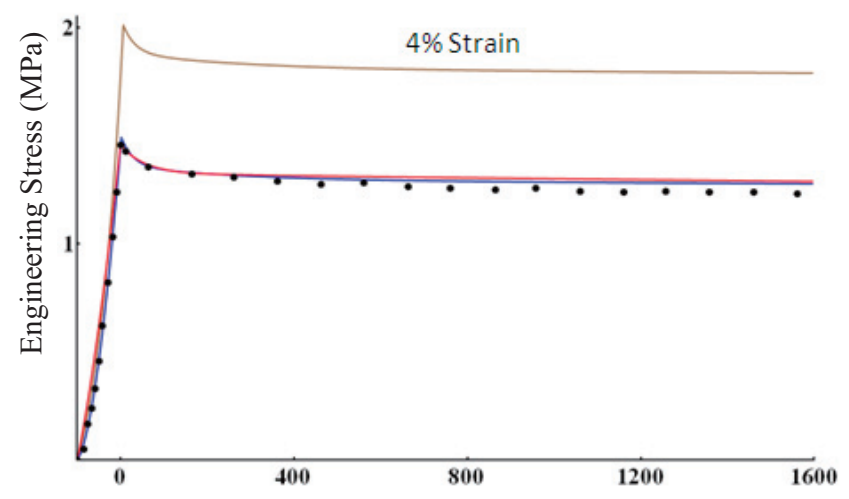

(b)

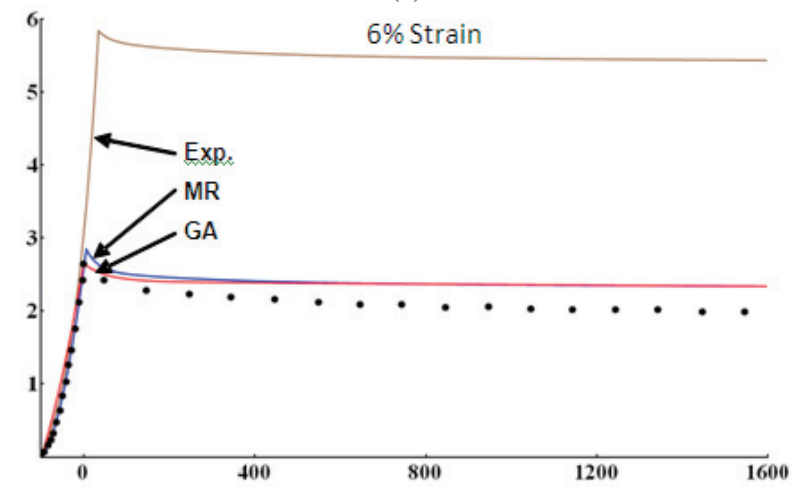

(c)

Fig. 1. Engineering stress versus time for the ACL, where the dots denote the experimental data.

During the prediction of the stress-relaxation response at the $4 \%$ strain (Fig. 1(b)), the MR model overestimates the experimental data by less than $2 \%$ and $6 \%$ at 100 s and $1600 \mathrm{~s}$, respectively, and the exponential model overestimates the stress response by $36 \%$ and $45 \%$ at 100 s and 1600s, respectively. The GA-based model shows a similar behavior to the MR model at 100s, but indicates more overestimation with up to $7 \%$ at 1600 s.

In case of predicting the stress relaxation at the $6 \%$ strain (Fig. 1(c)), the prediction of the overall stress response undergoes a great difference in correlation with the experimental data. On the change point of phases from 
ramping to relaxation (the peak stress point at $100 \mathrm{~s}$ ), the GA-based model overestimates by $1 \%$ compared to the overestimation of $3 \%$ obtained by the MR model. However, during the relaxation period of up to $1600 \mathrm{~s}$, the MR model predicts the stress response at a more decreasing rate than the GA-based model. The slow decrement of the GA-based model during the relaxation portion is monitored during several computational trials of GA. This trend is not unexpected and can be relatively adjusted when the focus on the specific part of experimental data is required. At the $6 \%$ strain level, the prediction of the exponential model is very poor and an overestimation of more than $100 \%$ is observed.

\section{CONCLUSIONS}

This paper studies the ability of GA to estimate and predict the QLV parameters of the ACL in order to define a systematic gradient-free bootstrapping alternative for intraoperative soft tissue characterization. Three different constitutive laws are used to describe the elastic stress response through both a gradient-based and gradient-free direct search method. Experiments and comparison analysis have been conducted to validate the approximation and prediction performance of the improved GA for the estimation of QLV parameters. Experimental results demonstrate that the proposed method can accurately estimate soft tissue paratemters in a time-saving process, and thus is suitable for intra-operative soft tissue characterization.

Future research will focus on establishment of an inverse solution for characterization of soft tissue properties from the experimental data obtained by in-situ robot-assisted measurements to predict the stress-strain behavior in response to different loading conditions such as cyclic stress relaxation and creep. Measurement of in-situ stress and strain poses extreme experimental difficulties, and these data cannot be obtained directly from the measured displacements and forces. A minimally invasive robotic indenter and associated analytical algorithms will be established for measurement of in-situ stress and strain.

\section{REFERENCES}

[1] C. Basdogan, S. De, J. Kim, M. Muniyandi, M. A. Srinivasan, "Haptic in minimally invasive surgical simulation and training", IEEE Comput. Graph. Appl., vol. 24, no. 2, pp56-64, 2004.

[2] Y. Zhong, B. Shirinzadeh, J. Smith, "Soft Tissue Deformation with Neural Dynamics for Surgery Simulation”, Int. J. Robot. Autom., vol. 22, no. 1, pp1-9, 2007.

[3] Y. Zhong, B. Shirinzadeh, G. Alici, J. Smith, "A Neural Network Methodology for Deformable Object Simulation”, IEEE T. Inf. Technol. B., vol. 10, no. 4, pp749-762, 2006.

[4] Y. Zhong, B. Shirinzadeh, G. Alici, J. Smith, "Simulation of Deformable Models with Poisson Equation", Comput. Method Biomec., vol. 9, no. 5, pp289-304, 2006.

[5] C. Basdogan, M. Sedef, M. Harders, S. Wesarg, "VR-based simulators for training in minimally invasive surgery", IEEE Comput. Graph. Appl., vol. 27, no. 2, pp57-66, 2007.

[6] E. Samur, M. Sedef, C. Basdogan, L. Avtan, O. Duzgun, "A robotic indenter for minimally invasive measurement and characterization of soft tissue response", Med. Image Anal., vol. 11, pp361-373, 2007.
[7] J. Kim, B. Ahn, S. De, M. A. Srinivasan, "An efficient soft tissue characterization algorithm from in-vivo indentation experiments for medical simulation", Int. J. Med Robot. Comp., vol. 4, pp277-285, 2008.

[8] S. L. Woo, G. A. Johnson, B. A. Smith, "Mathematical modeling of ligaments and tendons", J Biomech Eng-T ASME, vol. 115, pp468473, 1993.

[9] Y. C. Fung, Biomechanics: mechanical properties of living tissues. Springer: Berlin, Heidelberg, 1981.

[10] A. Chawla, S. Mukherjee, B. Karthikeyan, "Characterization of human passive muscles for impact loads using genetic algorithm and inverse finite element methods", Biomech. Model. Mechan., vol. 8, no. 1, pp67-76, 2009.

[11] H. Yamada, F. G. Evans, Strength of biological materials. Williams \& Wilkins: Baltimore, MD, 1970.

[12] B. Ahn, J. Kim, An Efficient soft tissue characterization under large deformations in medical simulations, Int. J. of Precis. Eng. and Manuf., vol. 10, no. 4, pp115-121, 2009.

[13] P. Provenzano, R. Lakes, T. Keenan, R. Jr Vanderby, "Nonlinear ligament viscoelasticity”, Annu. Rev. Biomed. Eng., vol. 29, pp908914, 2001.

[14] L. E. DeFrate, A. van der Ven, P. J. Boyer, T. J. Gill, G. Li, "The measurement of the variation in the surface strains of Achilles tendon grafts using imaging techniques”, J. Biomech. Eng-T ASME, vol. 39, pp399-405, 2006.

[15] L. E. DeFrate, G. Li, "The prediction of stress-relaxation of ligaments and tendons using the quasi-linear viscoelastic model", Biomech. Model Mechan., vol. 6, pp245-251, 2007.

[16] M. Mitchell, An introduction to genetic algorithms, MIT Press: Cambridge, USA, 1999.

[17] R. G. S. Asthana, "Evolutionary Algorithms and Nerual Networks", Soft Computing and Intelligent Systems: Theory and Applications, N. K. Sinha, L. A. Zadeh, M. M. Gupta (eds.), Academic Press: San Diego, USA, 2000.

[18] M. Kohandel, S. Sivaloganathan, G. Tenti, "Estimation of the quasilinear viscoelastic parameters using a genetic algorithm", Math. Comput. Model., vol. 47, pp266-270, 2008.

[19] D. P. Pioletti, L. R. Rakotomanana, J. F. Benvenuti, P. F. Leyvraz, "Viscoelastic constitutive law in large deformations: application to human knee ligaments and tendons", J. Biomech., vol. 31, pp753-757, 1998.

[20] G. A. Holzapfel, Nonlinear solid mechanics: a continuum approach for engineering, Wiley: New York, 2000.

[21] G. A. Johnson, G. A. Livesay, S. L. Y. Woo, K. R. Rajagopal, "A single integral finite strain viscoelastic model of ligaments and tendons", J Biomech Eng-T ASME, vol. 118, pp221-226, 1996.

[22] S. D. Abramowitch, S. L. Woo, "An Improved method to analyse the stress relaxation of ligaments following a finite ramp time based on the quasi-linear viscoelastic theory", J Biomech Eng-T ASME., vol. 126, pp92-97, 2004.

[23] J. A. Weiss, J. C. Gardiner, "Computational modeling of ligament mechanics”, Crit. Rev. Biomed. Eng., vol. 29, pp303-371, 2001.

[24] D. P. Pioletti, L. R. Rakotomanana, "On the independence of time and strain effects in the stress relaxation of ligaments and tendons", J. Biomech., vol. 33, pp1729-1732, 2000. 